\title{
Tumour necrosis factor superfamily cytokines and the pathogenesis of inflammatory osteolysis
}

\author{
J Lam, Y Abu-Amer, C A Nelson, D H Fremont, F P Ross, S L Teitelbaum
}

Ann Rheum Dis 2002;61(Suppl II):ii82-ii83

$\mathrm{P}$ eriarticular osteolysis is a debilitating complication of inflammatory arthritis. While controversy exists as to whether pannus, itself, is capable of degrading bone, the osteoclast is clearly the principal, if not exclusive, bone resorptive cell in rheumatoid disease and other osteopenic disorders. Thus, the treatment of inflammatory osteolysis depends upon understanding the means by which the osteoclast resorbs bone, and the mechanisms that govern its differentiation from precursor cells.

\section{OSTEOCLAST FUNCTION}

The osteoclast is a member of the monocyte/macrophage family and, in fact, macrophages from a variety of organs will commit to the osteoclast phenotype under appropriate culture conditions. ${ }^{1}$ This phenotype includes multinucleation, expression of markers such as the calcitonin receptor and tartrate resistant acid phosphatase and most importantly, the capacity to polarise when in contact with bone. The product of such polarisation is generation of the osteoclast ruffled membrane, which is a complex folding of the plasmalemma juxtaposed to bone matrix. The ruffled membrane is the resorptive organelle of the osteoclast and appears only when the cell is in the process of bone degradation. The means by which the osteoclast degrades bone involves its attachment to the target matrix, an event involving the $\alpha v \beta 3$ integrin. ${ }^{2}$ Once cell/matrix contact is extant, acidifying vesicles, within the osteoclast cytoplasm, move via microtubules, to the bone apposed plasma membrane $^{34}$ into which they insert by a process probably involving the small GTPase, Rab3. ${ }^{5}$ It is this insertion of acidifying vesicles into the plasmalemma that leads to its profound complexity thus generating the ruffled membrane. Moreover, this process endows the ruffled membrane with an abundance of vesicular proton pumps (H+-ATPases) that acidify an isolated microenvironment between the osteoclast and bone surface to a pH approximating 4.5. ${ }^{6}$ The acidification process mobilises bone mineral exposing the organic component of bone, largely type 1 collagen, which is subsequently degraded by the lysosomal enzyme, cathepsin $\mathrm{K}$, also a marker of the mature osteoclast. ${ }^{8}$

\section{OSTEOCLAST DIFFERENTIATION}

The past five years have witnessed great advances into understanding the mechanisms by which macrophages differentiate into osteoclasts. It had been appreciated for some time that induction of osteoclastogenesis, in vitro, requires contact of the osteoclast precursor with mesenchymal cells such as osteoblasts or their marrow stromal cell progenitors. Thus a stromal cell membrane-residing molecule dictates induction of the osteoclast phenotype. This molecule was shown, in 1997, to be receptor activator of NF- $\mathrm{KB}$ ligand (RANKL), a member of the tumour necrosis factor (TNF) superfamily, ${ }^{9}$ which is also expressed in cells of the immune system and breast. In fact, osteoclasts can now be generated, from macrophages, in the presence of only RANKL and M-CSF, the latter promoting survival and proliferation of the precursor. ${ }^{10}$

\section{THE TNF SUPERFAMILY AND THE OSTEOCLAST}

The local bone loss attending rheumatoid arthritis is associated with a plethora of inflammatory cytokines, among the most prominent being TNF $\alpha$. This being the case, we asked if TNF $\alpha$ is osteoclastogenic and if so, could we detail the mechanism by which the cytokine commits macrophages to the osteoclast phenotype. Our initial experiments involved the use of lipopolysacharide (LPS) a potent inducer of TNF $\alpha$ expression. ${ }^{11}$ We find that LPS induces osteoclastogenesis, in vitro, and does so through a process involving the $\mathrm{p} 55$, but not p75 TNF receptor. Mirroring these in vitro studies, LPS is a potent osteoclastogenic agent in wild type mice but does not affect those lacking the p55 TNF receptor. This finding is in keeping with subsequent experiments establishing that TNF $\alpha$, in itself, induces osteoclast differentiation in vitro and in vivo. ${ }^{10}$ Interestingly and in contrast to $\mathrm{p} 55 \mathrm{TNF}$ receptor, the p75 receptor, which recognises primarily the membrane bound form of the cytokine, is anti-osteoclastogenic. ${ }^{12}$

While these experiments validate that the osteoclastogenic effects of TNF $\alpha$ are mediated via its $\mathrm{p} 55$ receptor, they do not establish the target cell of the cytokine. Specifically, the two cell types essential for authentic osteoclastogenesis-namely, the macrophage and the marrow stromal cell or its derivatives, each express TNF receptors. Thus, either cell type (or both) may theoretically transmit the primary TNF signal. The potential role of stromal cells as the target is buttressed by the fact that TNF $\alpha$ increases expression of the key osteoclastogenic cytokine, RANKL, by these cells. Alternatively, the possibility existed that TNF, independent of RANKL would directly stimulate macrophages to undergo osteoclastogenesis. This second hypothesis was held by a number of previous reports.

We investigated this issue by affinity purifying osteoclast precursors in the form of bone marrow macrophages to greater than $99 \%$ homogeneity. ${ }^{10}$ Regardless of the concentration of TNF $\alpha$ to which these cells are exposed, they fail to differentiate into osteoclasts. On the other hand, addition of less than one percent of RANKL sufficient to induce osteoclastogenesis to minuscule amounts of TNF $\alpha$ prompts exuberant osteoclast differentiation. This synergy of the two cytokines is reflected by a similar effect on activation of the downstream signalling molecule, NF- $\mathrm{KB}$, an essential osteoclastogenic transcription complex. These experiments led us to postulate that while RANKL is necessary for TNF $\alpha$ induced osteoclastogenesis, the former cytokine need not be present in more than constitutive levels. To investigate this issue, in vivo, we generated chimeric mice consisting of bone marrow macrophages responsive to TNF $\alpha$ while their stromal cells are not. ${ }^{10}$ The marrow stromal cells were therefore capable of expressing only basal, non-induced quantities of RANKL despite the presence of TNF $\alpha$. Confirming that basal, non-induced

Abbreviations: TNF, tumour necrosis factor; LPS, lipopolysacharide 


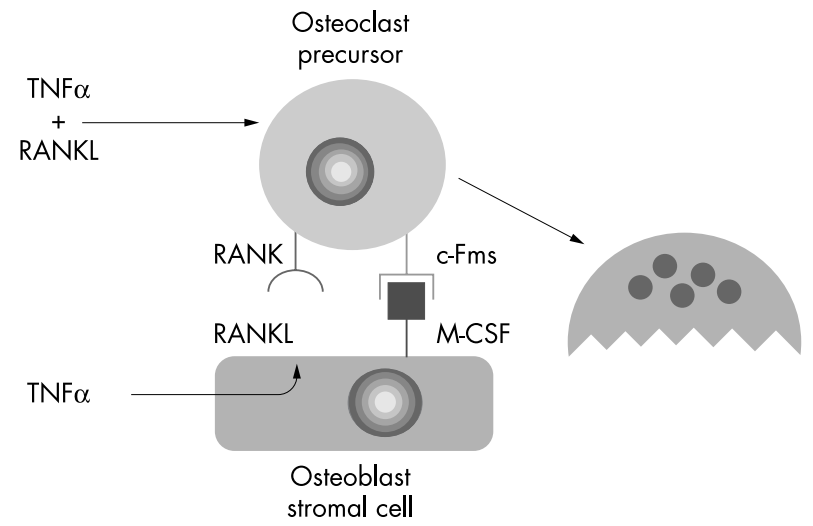

Figure 1 TNF $\alpha$ mediated osteoclastogenesis requires RANKL. TNF $\alpha$ targets two cells in the osteoclastogenic process. In the first instance the cytokine induces RANKL expression by stromal cells. Increased RANKL interacting with its receptor RANK, in a cell contact dependent manner, stimulates osteoclast differentiation. TNF $\alpha$ also directly provokes osteoclast precursors to differentiate, but this event requires permissive levels of RANKL.

amounts of RANKL allow TNF to enhance osteoclast differentiation, administration of TNF $\alpha$ to these chimeric animals prompts osteoclastogenesis at a level commensurate with that seen in wild-type animals receiving the cytokine. The critical message of these studies is that although TNF $\alpha$ alone cannot induce osteoclast differentiation, it is very effective in the presence of permissive levels of RANKL

\section{CRYSTAL STRUCTURE OF RANKL}

Interestingly, RANKL is a member of the TNF superfamily and shares much homology with TNF $\alpha$. The fact, however, that only RANKL can engage RANK and thereby induce osteoclast differentiation ${ }^{10}$ indicates that it possesses unique structural features. To investigate this issue, we solved the structure of RANKL to a resolution of 2.6 angstroms by $x$ ray crystallography. ${ }^{13}$ Like other members of the TNF superfamily, RANKL monomers assume a prototypical "jellyroll" topology and spontaneously associate as homotrimers. While the $\beta$-strand core scaffold of RANKL is similar to that of other TNF cytokines, the sovent-accessible loops connecting these $\beta$-strands are unique in residue composition and surface topology. The significance of this observation lies in the fact that it is the unique surface elements of the TNF family cytokines that underlie receptor-binding specificity. Given this fact, and the structrual congruence of the ligand binding domains of DR5 and the p55 TNF receptor, we modelled the probable receptor contact points on RANKL by superimposition of these two TNF superfamily receptors. This exercise showed that prospective RANK contact sites, on RANKL, are structurally unique in comparison with other TNF cytokines, encompassing residues in the $\mathrm{AA}^{\prime \prime}, \mathrm{CD}$, and $\mathrm{DE}$ loops. To determine if these putative RANK contact sites on RANKL endow the cytokine with its unique osteoclastogenic properties, we performed structure based mutagenesis experiments. Specifically, we (1) replaced Ile248 with Asp in the DE loop, (2) deleted most of the $\mathrm{AA}^{\prime \prime}$ loop, and (3) replaced the $\mathrm{AA}^{\prime \prime}$ loop of RANKL with that of TNF. Confirming our hypothesis that the mutated structures engender the capacity of RANKL to promote osteoclast differentiation, deletion or TNF swap of the AA" loop completely abrogates osteoclastogenesis and the $\mathrm{DE}$ loop point mutation dampens the process eightfold. Thus, $\mathrm{TNF} \alpha$ is clearly central to the pathogenesis of inflammatory osteolysis but it requires the presence of RANKL. While both cytokines are homotrimeric members of the same superfamily, RANKL contains distinct structural features which explain its unique osteoclastogenic properties.

\section{Authors' affiliations}

J Lam, Y Abu-Amer, C A Nelson, D H Fremont, F P Ross, S L Teitelbaum, Washington University School of Medicine, St Louis, USA

Correspondence to: Dr S L Teitelbaum, Barnes-Jewish Hospital, MS 90-31-649, 216 S Kingshighway, St Louis, MO 63110, USA; teitelbs@medicine.wustl.edu

\section{REFERENCES}

1 Teitelbaum SL. Bone resorption by osteoclasts. Science 2000;289:1504-8.

2 McHugh KP, Hodivala-Dilke K, Zheng MH, Namba N, Lam J, Novack $D$, et al. Mice lacking $\beta_{3}$ integrins are osteosclerotic due to dysfunctional osteoclasts. J Clin Invest 2000; 105:433-40

3 Blair HC, Teitelbaum SL, Ghiselli R, Gluck S. Osteoclastic bone resorption by a polarized vacuolar proton pump. Science 1989;245:855-7.

4 Abu-Amer Y, Ross FP, Schlesinger P, Tondravi MM, Teitelbaum SL. Substrate recognition by osteoclast precursors induces c-src/microtubule association. J Cell Biol 1997; 137:247-58.

5 Abu-Amer Y, Teitelbaum SL, Schlesinger P, Ross FP. Expression and regulation of RAB3 proteins in osteoclasts and their precursors. J Bone Miner Res 1999; 14:1855-60.

6 Silver IA, Murrills RJ, Etherington DJ. Microelectrode studies on the acid microenvironment beneath adherent macrophages and osteoclasts. Exp Cell Res 1988I; 175:266.

7 Blair HC, Kahn A, Crouch EC, Jeffrey JJ, Teitelbaum SL. Isolated osteoclasts resorb the organic and inorganic components of bone. J Cell Biol 1986;102:1164-72.

8 Li YP, Chen W, Liang Y, Li E, Stashenko P. Atp6i-deficient mice exhibit severe osteopetrosis due to loss of osteoclast-mediated extracellular acidification. Nature Genet 1999;23:447-51.

9 Lacey DL, Timms E, Tan H-L, Kelley M, Dunstan CR, Burgess T, et al. Osteoprotegerin ligand is a cytokine that regulates osteoclast differentiation and activation. Cell 1998;93:165-76.

10 Lam J, Takeshita S, Barker JE, Kanagawa O, Ross FP, Teitelbaum SL. TNF $\alpha$-induces osteoclastogenesis by direct stimulation of macrophages exposed to permissive levels of RANK ligand. J Clin Invest 2000; 106:1481-8

11 Abu-Amer Y, Ross FP, Edwards J, Teitelbaum SL. Lipopolysaccharide-stimulated osteoclastogenesis is mediated by tumor necrosis factor via its p55 receptor. J Clin Invest 1997; 100:1557-65.

12 Abu-Amer Y, Erdmann J, Kollias G, Ross FP, Teitelbaum SL. Tumor necrosis factor receptors types 1 and 2 differentially regulate osteoclastogenesis. J Biol Chem 2000;275:27307-10.

13 Lam J, Nelson CA, Ross FP, Teitelbaum SL, Fremont DL. Crystal structure of the TRANCE/RANKL cytokine reveals determinants of receptor-ligand specificity. J Clin Invest 2001;108:971-9. 